\title{
Vision controlled robotic furniture system
}

\author{
Christos Georgoulas *, Thomas Linner, and Thomas Bock \\ Department of Architecture, Building Realization and Robotics Lab, Technical University \\ Munich, Germany \\ *Corresponding author (christos.georgoulas@br2.ar.tum.de)
}

\begin{abstract}
Purpose Ergonomics is the engineering science that is concerned with the physical and psychological relationship between machines and the people who use them. The key is to get the best out of something with the smallest possible effort. In ergonomics function is as important as aesthetics. Ergonomics is concerned with the creation of a product or an environment, where the connection between human skills and the surroundings is optimized. The aim of the proposed paper is the implementation of an ergonomic furniture system concentrating on height adjustments in particular. Method Furniture is designed to adapt to specific needs, supporting various human activities. According to the application, a set of guidelines or rules is followed during design in order to provide ergonomic features. Small or large sized furniture can be found within the household, comprising individual modules, placed at a specific height, based upon the desired functionality. In order to realize the proposed concept, a development approach was followed. First, thorough research was conducted to identify the actual need of such a system and define the appropriate design specifications. According to the various existing technologies and processes, the authors identified those that met the design requirements, and those better adapted to the proposed system. Once the initial concept was finalized, a series of experiments were carried out in a real environment, implementing the prototype of the proposed system. Results \& Discussion The proposed robotic furniture system provides an integrated solution, consisting of reduced space utilization, modularity, and intelligent operation, while respecting ergonomic principles. A set of electrical motors was used to position the various sections of the system at the correct height level, and to displace them on the horizontal axis to allow a rotational motion path. The implemented prototype was evaluated in terms of ergonomics (using an age simulation suite), organizational ergonomics within a room, and space utilization (as the individual sections/shelves can rotate in a vertical direction, the proposed system can be installed in small rooms, achieving $20 \%$ space utilization efficiency providing more space for mobility with walking frames or wheelchairs). A vision system was integrated into the prototype to perform object recognition-for efficient classification of objects stored into the various system sections, for assistance in retrieving a specific previously stored object by moving the appropriate section of the furniture up or down to the correct height. The real-time response of the vision system, efficiently addresses the need for a short time delay between user queries and system response. The robotic furniture system also serves as a lift, a seat, or a working place for elderly people, i.e. it efficiently applies ergonomics to issues in the living environment of the ageing society. Fusing functions and services such as infotainment or internet connectivity, also provides extended functionality to the user. Many everyday activities can be dealt with a more efficiently from a single terminal.
\end{abstract}

Keywords: ambient integrated robotics, ergonomic furniture, vision system, architecture

\section{INTRODUCTION}

A close look at the fixed and loose components of our environments shows that all components of the housing environment are gradually integrated with electronics and micro-systems. "Miniaturization" and "Downscale" as basic trends of our technological development today enable a seamless integration of sensors, actuators, control components and microelectronic systems into all subsystems, components and appliances, in a pervasive but somehow invisible way.

Today, robots and distributed robotic sub-systems start to permeate our every day surrounding, enhancing it with services and additional features. At the same time, this permeation is on the way to transform our perception of what robots are, robot technology, robots' possibilities and the environment they are merged with. This transformation which has to be understood as a natural part of the evolution of robotics, will especially become visible when robots enter the field of service and assistance.

Ageing society faces numerous challenges in performing simple tasks in Activities of Daily Living $(A D L s)^{3}$. ADLs represent the everyday tasks people usually need to be able to independently accomplish. An environment populated with robotic elements and micro-systems can undoubtedly contribute in enhancing their independence, by introducing a degree of ambient assistance. It must be noted though that populating a home environment with robotic elements must be performed following a space-efficient utilization scheme. Elderly people, and especially the ones using assistive devices such as wheelchairs and rollators, require increased barrier-free space for mobility purposes. Therefore, the following article describes the design and implementation of a novel 
robotic furniture system which also serves as a lift or seat, storage space, health assistance terminal, and working place for elderly people.

Additionally, an integrated vision system implements the human-machine interaction assisting in detecting stored items in the robotic furniture, and aiding in efficiently retrieving them upon user queries issued by vocal commands. Thus, a compact terminal assisting the ageing society in ADLs by enhancing their independence, as well as offering extended ergonomy is proposed.

\section{RESEARCH AND SURVEY}

Some researchers already proposed integrated solutions as e.g. Robotic Rooms ${ }^{4}$, Wabot House ${ }^{5}$, or Robot Town ${ }^{6}$. The aim of those approaches was to distribute sensors and actuators in the environment which can communicate with the intended robot system, allowing simpler and robust robot designs. The main objective of these approaches was to assist humans in daily living by distributing mechatronic devices within environments to allow a humanmachine interaction providing a series of services and applications.

However, these approaches integrate mainly sensors, actuators and robots on an informational level. Furthermore, they are presenting implementations that are realized in a controlled experimental environment, and cannot be straightforwardly applied into a regular medium sized apartment. The proposed system focuses on providing a compact ergonomic system that can be easily installed within a home environment, without requiring major rearrangement procedures and modifications.

The required $\mathrm{m}^{2}$ space ratio per person is rising continuously. Nowadays, according to national surveys, an average person requires approximately 45 $\mathrm{m}^{2}$. Since the 1990s this figure has raised nearly by $10 \mathrm{~m}^{2}$. Currently it rises about $0.55 \mathrm{~m}^{2}$ per year. It is estimated that by $2030,55 \mathrm{~m}^{2}$ will be required per person. At the same time real estate prices in major cities around the world are skyrocketing. Thus, space utilization is becoming an important parameter when proposing cost-effective solutions within the home environment. The proposed approach issues a combination of organization, design and technology, in order to simplify ADLs while maintain minimization of space utilization requirements.

\section{Ergonomy}

Ergonomy is the science of designing user interaction with equipment and workplaces to fit the user. It is all about to get the best of something, but with the smallest effort. Because of the 'ergonomics' the sense of function is as much important as the sense of aesthetics. Ergonomics is about creating a product or an environment, where the connection between human skills and the surrounding is optimized.
First studies about ergonomy were made in the USA during the industrialization period to optimize the efficiency of the workers. Later on, there were more studies during the world wars to examine efficiency of new and complex machines and weaponry.

More researches were made at the BauHaus, a school in Germany that combined crafts and fine arts, and was famous for the approach to design that it publicized and taught. Henry Dreyfuss, one of the celebrity industrial designers of the 1930s and 1940s, was the first who had really consequently studied and also used ergonomy in his design studio. He just did not care about the outward appearance of his products but he also cared about how to use them in a good way.

Outside of the discipline itself, the term 'ergonomics' is generally used to refer to physical ergonomics as it relates to the workplace (as in for example ergonomic chairs and keyboards). Ergonomics in the workplace has to do largely with the safety of employees, both long and short-term. Workplaces may either take the reactive or proactive approach when applying ergonomics practices. Reactive ergonomics is when something needs to be fixed, and corrective action is taken. Proactive ergonomics is the process of seeking areas that could be improved and fixing the issues before they become a large problem. Problems may be fixed through equipment design, task design, or environmental design. Equipment design changes the actual, physical devices used by people. Task design changes what people do with the equipment. Environmental design changes the environment in which people work, but not the physical equipment they use.
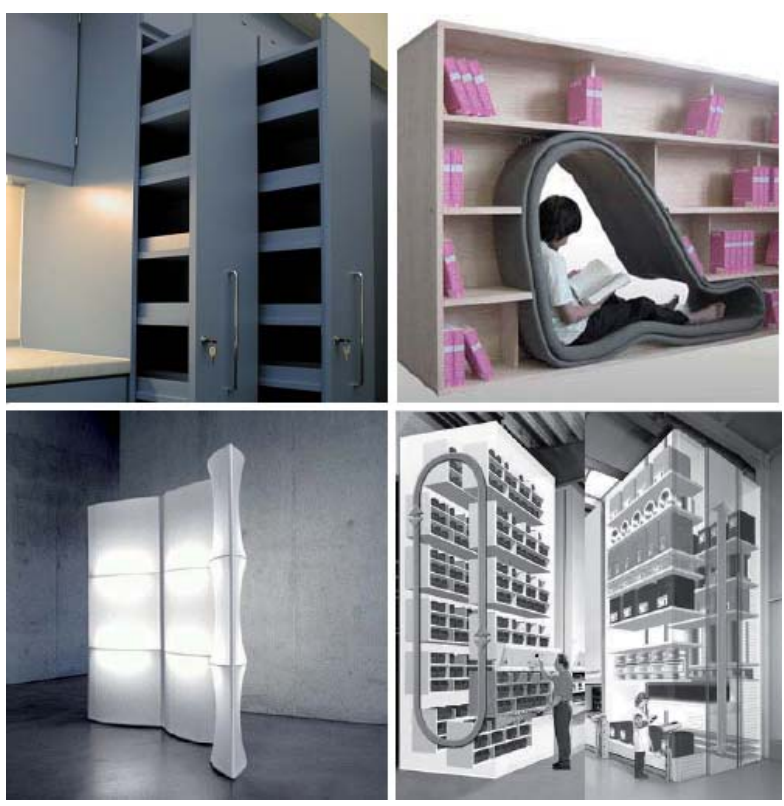

Fig.1. Applied ergonomy 


\section{Current Standards and Problems}

A research was performed in order to identify ergonomy in ageing society environments. Elderly people usually apply some degree of ergonomy, by adapting their environment in a way to serve their needs. Thus they usually accumulate less frequently or unwanted items in high, out of reach levels, and more frequently used items in their proximal low level area. Figures 2 and 3 present this observed behavior.

Storage space is, especially for elderly people, a very important requirement, since they accumulate over time a lot of personal items. This occurs due to the fact that very often, items that are forgotten, or not found, are replaced by new ones, resulting thus in excessive storage space loss. It is necessary to improve the accessibility to such items in order to enhance the ability of retrieval and reduce redundancy in storage space utilization.

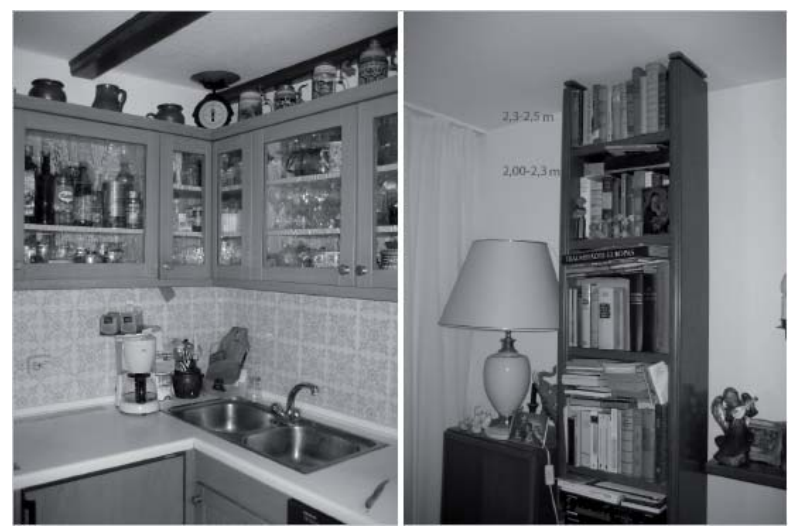

Fig.2. Unused items stored in high, out of reach levels

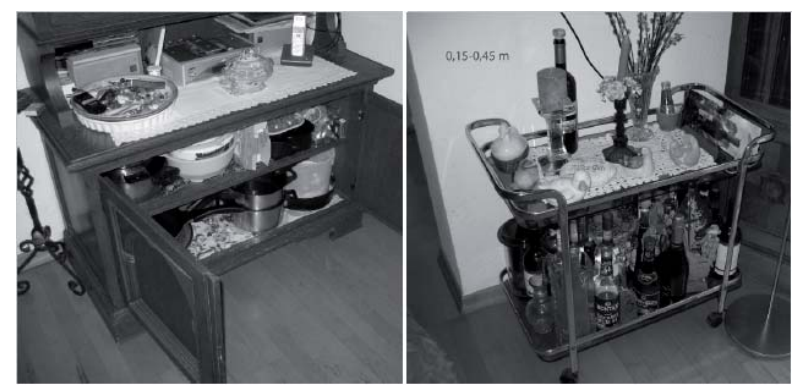

Fig.3. Accumulation of frequently used items within the proximity and relatively low levels

\section{Proposed Concept}

In order to efficiently deal on assisting elderly people in retrieving stored items a robotic furniture system is proposed. The conception of the design was influenced by observing the arrangements elderly people follow in their home environment in order provide them with direct access to their frequently used items. Space redundancy is limited by the proposed design approach since the robotic furniture introduces a height adjustable storage solution. A set of electrical motors is used to position the various sections of the system into the correct height level, and to displace them towards the horizontal axis, to allow a rotational motion path, Figure 4.

Elderly people accumulate items they frequently require in their proximal area, due to the fact that they want to allow comfortable and direct access to them, form a sitting posture. Even though the ideal level of storing items is in places that they can be easily retrieved form a standing or sitting position, i.e. between 0.5 to $1.5 \mathrm{~m}$, due to storage space absence, they tend to store items in low level places as well, where they can be not so straightforwardly reached. This introduces some limitations since elderly people face stiffness issues and disabilities. Furniture offering storage space that can be adjusted in height, significantly contributes to overcome such limitations, while offering extreme functionality and ergonomy, utilizing less space compared to conventional storage furniture.

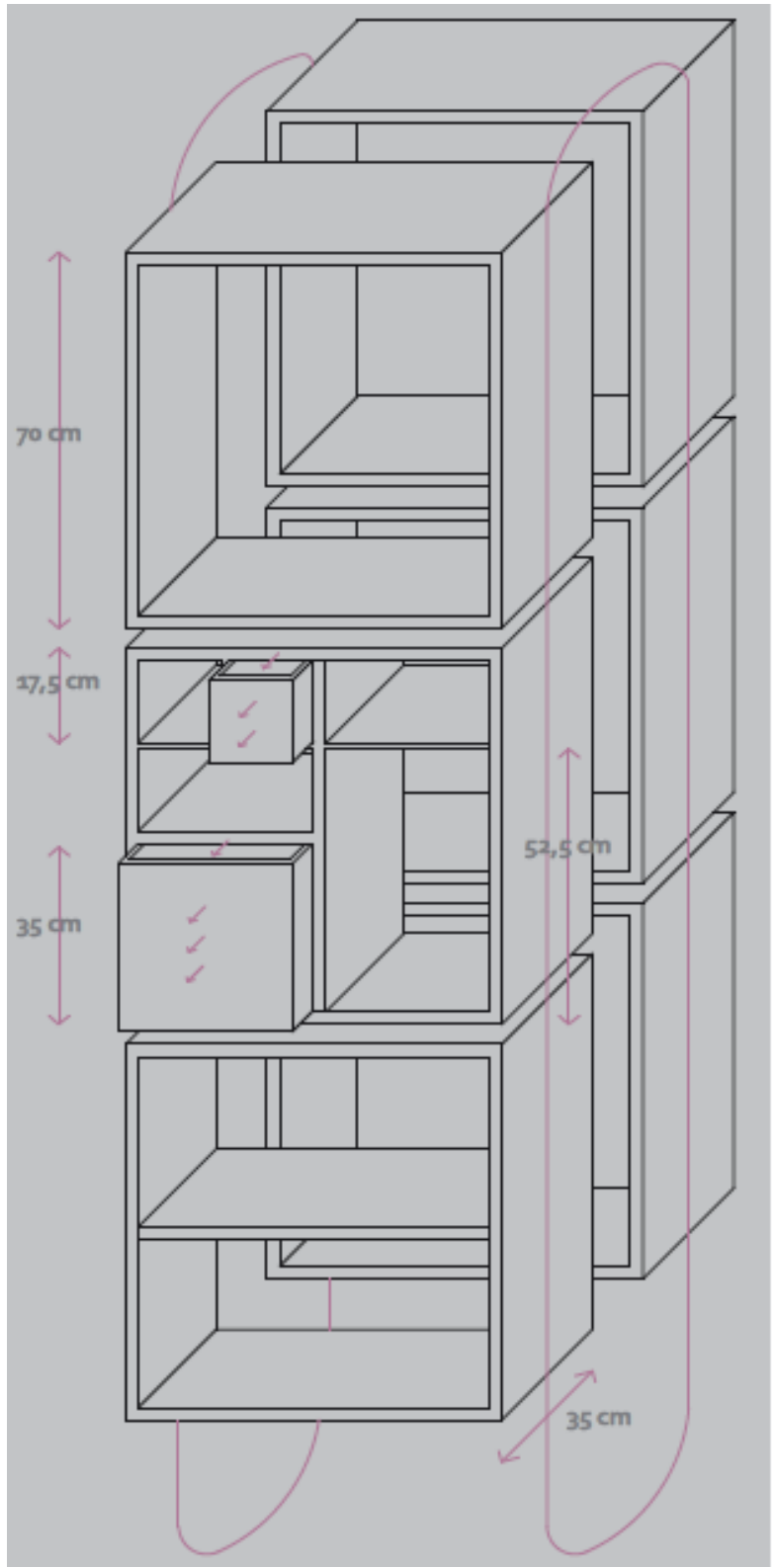

Fig.4. Rotary Motion Path

The idea is based on adaptive and space-dividing furniture, offering vertical height adjustment, accord- 
ing to the user needs. The proposed concept was also considered to be applied in various areas within a home environment. The system can be manufactured and customized according to the required application, such as apartment entrance, living room, kitchen, bathroom, etc. Furthermore, since the robotic furniture is accessible on sides, front and back, it can be used to partition space in an efficient way, introducing a barrier-free approach, assisting in the mobility of elderly people using wheelchairs and rollators, and requiring less space utilization for installation, Figure 5. A saving of up to $20 \%$ is estimated compared to a conventional apartment arrangement, since all required functions such as beds, sofas, tables, chairs, etc, can be integrated into the proposed concept, deployed upon request using robotic actuators and sensors, Figure 6.
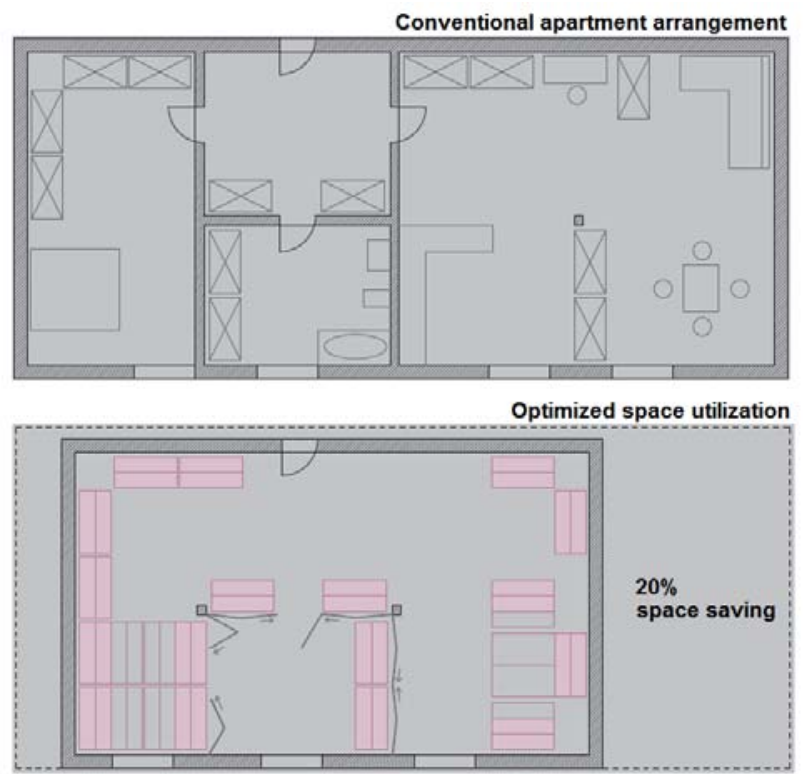

Fig.5. Barrier-free design approach offering enhanced space saving

\section{SOLUTION APPROACH}

Once conceiving the proposed concept, it was then realized in a 1:1 scale partially functioning prototype to allow evaluation and testing, Figure 7.

A set of motorized servos arrangement is going to be later added to the prototype for the vertical and horizontal displacement of the robotic wall elements, controlled by a microprocessor. Each element then can be transported via a chain type belt, attached to the servo gears. A grid of rail type mechanisms is already implemented to secure the elements and allow a smooth vertical and horizontal translation of each one, leading to a rotational motion path, Figure 8.

\section{Evaluation}

The prototype was evaluated in terms of:
- Ergonomy (Using and Age Simulation suite)

- Organizational Ergonomy within a room

- Space Use (as the individual sections/shelves can rotate it is possible to use rooms more in a vertical direction and about $20 \%$ space for storage on the ground can be saved and generate free space for mobility with rollators or wheelchairs)

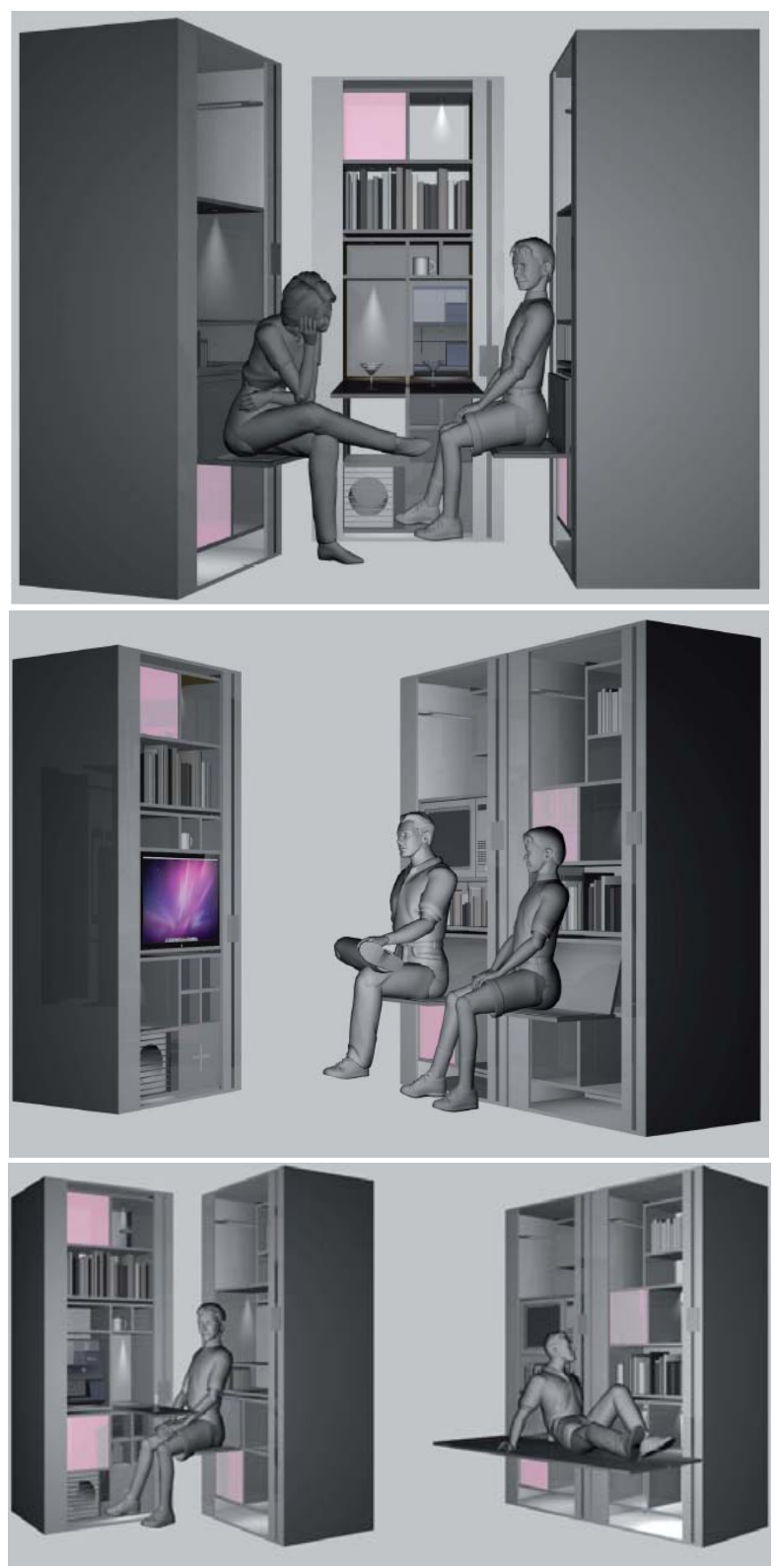

Fig.6. Proposed concept configurations

The evaluation using an age simulation suite was performed, Figure 9, in order to simulate the limitations elderly people face in performing simple movements. In order a furniture to provide ergonomic features, it must be designed and manufactured according to a set of standards that would allow unobtrusive access, optimum space utilization and adaptability to the user needs. The evaluation procedure revealed that the proposed furniture design allows direct access, addresses elderly people mo- 
bility problems, and enables space utilization saving while maintaining improved functionality.

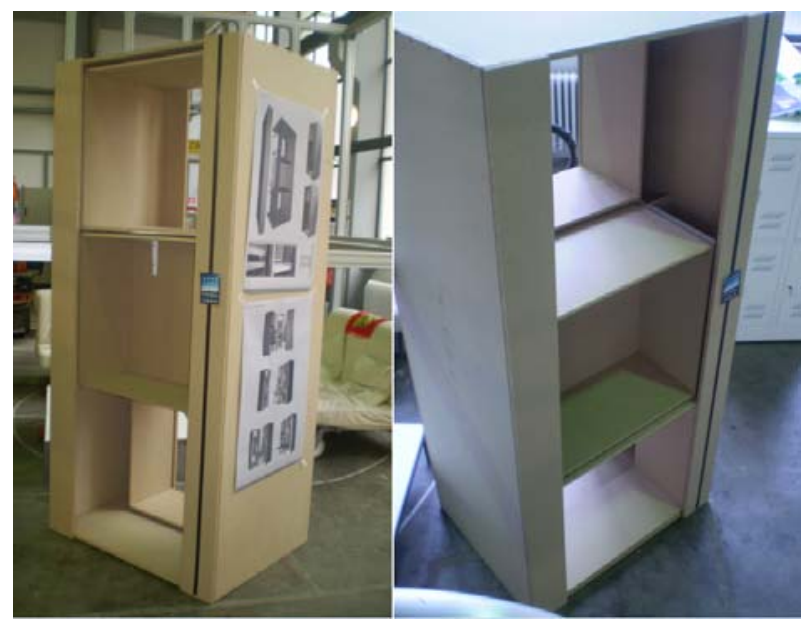

Fig.7. 1:1 scale implemented prototype

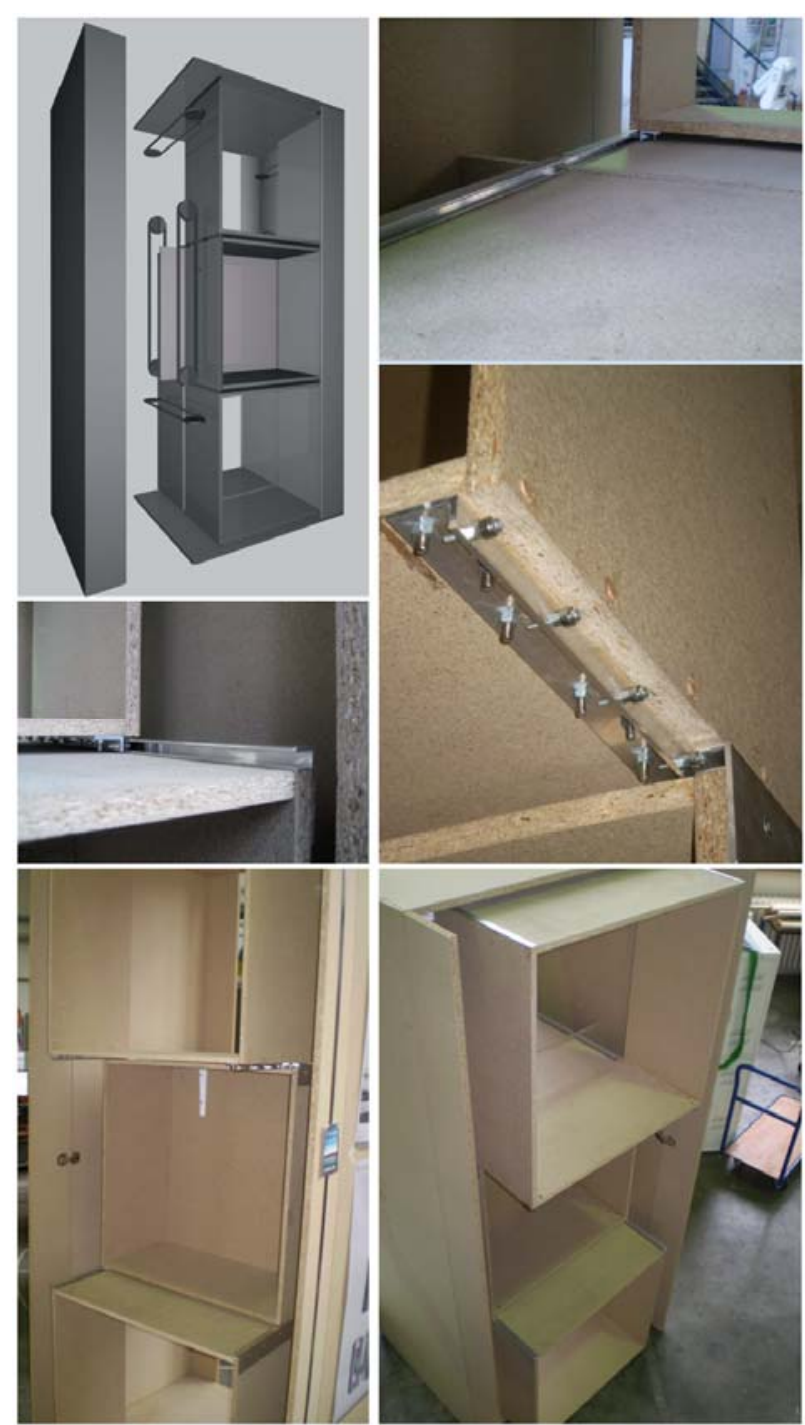

Fig.8. Rotational and translational mechanisms

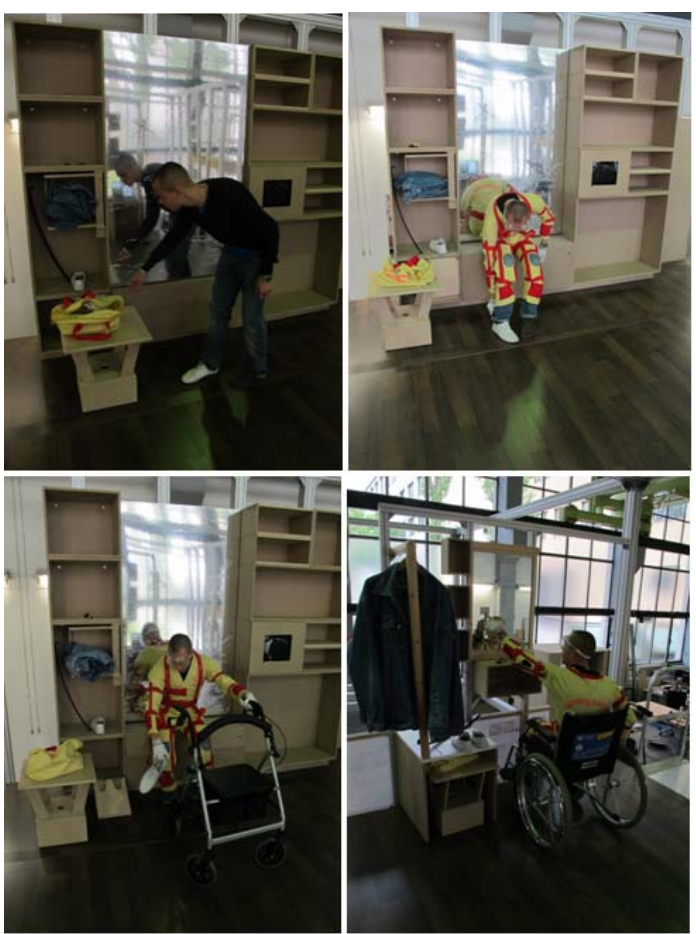

Fig.9. Evaluation of the robotic furniture system by an age simulation suite

\section{Proposed System FrameWORK}

The proposed system architecture is depicted in Figure 10. The human-machine interaction is performed via visual and vocal information exchange between the user and the robotic furniture.

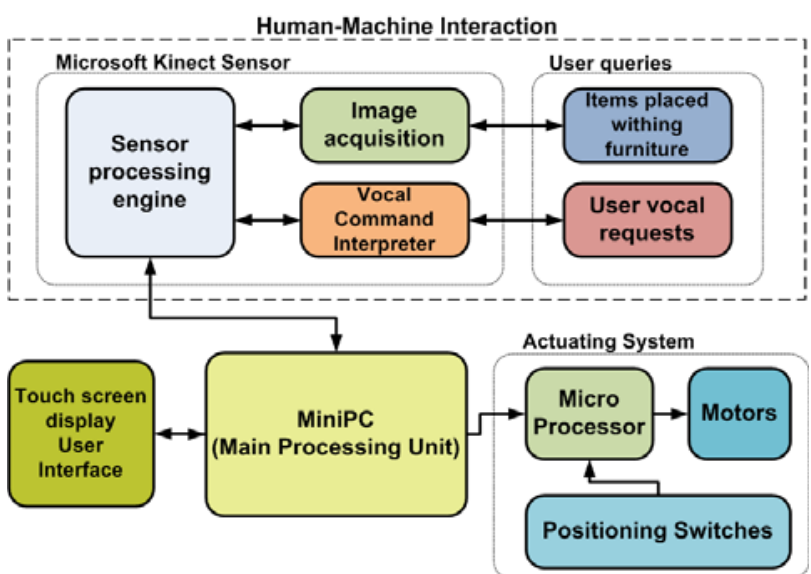

Fig.10. Block diagram of the proposed system framework

A miniPC is used to serve as the main processing unit, interfaced with a Microsoft Kinect Sensor ${ }^{7}$, an Arduino-based ${ }^{8}$ microcontroller board driving the motors of the robotic furniture, and a touch screen display which provides an appropriate graphical user interface (GUI) to allow efficient operation of the system.

The user stores and retrieves items via the middle shelf of the robotic furniture. The Kinect Sensor acquires color RGB $640 \times 480$ pixels spatial resolution images, of the stored items within the middle shelf. 
For evaluation purposes items with different colors were used. Different color items were placed into different shelves of the robotic furniture. In order for the user to retrieve an item, a vocal command is issued corresponding to the required item color. The vocal interpreted is implemented using Microsoft Kinect embedded microphone, which provides voice recognition capabilities. The position control mechanism issues a command to position the correct shelf in the middle position of the furniture in order for the user to retrieve the required item.

The application was programmed using C\# programming language, for interfacing the Arduino motor controller board, Microsoft Kinect Sensor, and implementing the required graphical user interface projected to the touch screen display.

\section{Results AND Future DeVElopment}

The proposed vision controlled robotic furniture is still under development in order to define final specifications and offered services, and to enhance its functionality and operation towards the user.

Currently the vocal user queries are limited to voice commands representing various colors. The vision system acquires an RGB image which is processed and the corresponding item color is extracted, in order to classify the item position in the corresponding robotic furniture shelf. The proposed study aims to integrate a more intelligent visual algorithm, which would classify objects not only according to their color, but to their identity using an object recognition algorithm. Additionally, the Kinect visual sensor is able to acquire depth information, allowing thus multiple items stored in the same shelf to be efficiently detected and identified.

Tests implemented to evaluate system performance presented a real-time response between user queries and resulting signaling commands to the motor drives. Since the tests were conducted by observing the corresponding output signals issued by the Arduino microcontroller board, that are going to be later interfaced to the electrical motor drives, the observed real-time response, efficiently provides a responsive system with minimal error rate. Errors are currently introduced by the voice recognition module, since vocal color commands are sometimes interpreted into the wrong color. This error rate can be eliminated by an appropriate voice recognition training stage, corresponding to a new set of vocal commands that would allow efficient differentiation of user queries.

Additional features such as health monitoring sensors, medication dispenser, deployable seats and tables, are currently considered to be integrated into the robotic furniture. Such an approach can offer an improved service delivery environment, by utilizing a compact and ergonomic furniture terminal, based upon advances in sensors and sensor networks, robotic elements, mechatronic devices, pervasive computing, and artificial intelligence.

\section{Conclusions}

The proposed concept presented in Figure 6, aims in creating a customized environment using several configurations of novel visually controlled robotic furniture system, providing enhanced services to the ageing society, while maintaining ergonomy. Service delivery is important to elderly people since it increases their independence, allows them to remain longer in their homes, and improves their living quality. By distributing intelligent robotic elements within the home environment, daily human life is revolutionized by making people's surroundings flexible and adaptive. In the proposed research, technologies are deployed to make computers disappear in the background, while the human moves into the foreground in complete control of the augmented environment. The implemented prototype was evaluated in terms of, ergonomy (using an age simulation suite), organizational ergonomy within a room, and space utilization (as the individual sections/shelves can rotate in a vertical direction, the proposed system is possible to be installed in small rooms, achieving a $20 \%$ less space utilization usage, in order to allow free space for mobility with rollators or wheelchairs). A vision system integrated on the prototype to perform object recognition, efficiently classifies objects stored into the various system elements, in order to assist the user in retrieving a specific previously stored object, by moving the appropriate section of the furniture into the correct height. The real-time response of the vision system, efficiently addresses the need for short time delay between user queries and system response.

The robotic furniture system can also serves as a lift or seat, or working place for elderly people, efficiently addressing the ageing society issues in living environments by providing extended ergonomy. Fusing functions and services such as infotainment or internet connectivity, also provides extended functionality to the user, once many everyday activities can be dealt with a more efficient way, all from a single terminal.

\section{References}

1. Intille, S., Larson, K., Tapia, E.M., "Designing and evaluating technology for independent aging in the home", Proceedings of the International Conference on aging, disability and independence, 2003.

2. Frintrop, S., "VOCUS: A visual attention system for object detection and goal-directed search", Ph.D. dissertation, Bonn, Germany, July 2005, Lecture Notes in Artificial Intelligence (LNAI), Vol.3899, Springer Verlag, 2006.

3. Wiener, J.M., Hanley, R.J., Clark, R., Van Nostra, J.F., "Measuring the activities of daily living: com- 
parisons across national surveys", Journal of Gerontology, Social Sciences, Vol. 46, pp. 229-237, 1990.

4. Sato, T., Harada, T., Mori, T., "Environment-type robot system "RoboticRoom" featured by behavior media, behavior contents, and behavior adaptation", IEEE Transactions on Mechatronics, Vol. 9 (3), pp. $529-534,2004$.

5. Waseda University, "Wabot House", http://www.wabot-house.waseda.ac.jp/html/etop.htm

6. Murakami, K., Hasegawa, T., Karazume, R., Kimuro, Y., "A Structured Environment with Sensor Networks for Intelligent Robots", IEEE Sensors, pp. 705-708, 2008.

7. Microsoft Corp. Redmond WA., "Kinect Xbox 360", www.microsoft.com.

8. Arduino open source platform, "Arduino", http://www.arduino.cc/ 